\title{
Cancers Tumor Detection using Magnetic Resonance Imaging with Ant Colony Algorithm
}

\author{
Uttamjeet Kaur $^{1}$, Rajesh Sharma ${ }^{2}$, Mandeep Dosanjh ${ }^{3}$ \\ CSE, RIET Phagwara, Punjab, India ${ }^{1,2,3}$
}

\begin{abstract}
The most fatal disease in the era of medical science is the brain tumor.it can be diagnosed if detected at the early stage of the tumor growth. Various techniques are developed \& has been applied on magnetic resonance imaging to detect the abnormality in the brain as tumor. The segmentation of these magnetic resonance images is very difficult then natural images.
\end{abstract}

Key Words: Multi-Thresholding, Brain Magnetic Resonance Imaging, Ant Colony Optimization.

\section{INTRODUCTION}

Medical imaging is often perceived to designate the set of techniques that noninvasively produce images of the internal aspect of the body. In this restricted sense, medical imaging can be seen as the solution of mathematical inverse problems. This means that cause (the properties of living tissue) is inferred from effect (the observed signal). In the case of ultrasonography the probe consists of ultrasonic pressure waves and echoes inside the tissue show the internal structure. In the case of projection radiography, the probe is X-ray radiation which is absorbed at different rates in different tissue types such as bone, muscle and fat. The biomedical imaging's scope covers image reconstruction, data acquisition, and image analysis, methods, systems, involving theories, and applications. While post-processing and tomography techniques become increasingly advanced, traditional and emerging techniques play more and more crucial roles in anatomical, molecular, cellular and functional imaging. Medical imaging is the technique and process used to create images of the human body and structures for clinical purposes (medical processes looking to unveil, diagnose, or examine disease) or medical science (involving the study of basic anatomy and physiology).

Although for medical reasons imaging of removed organs and tissues can be performed, but such procedures are usually referred to as part of pathology rather than medical imaging. In its widest sense and as a discipline, it is part of biological imaging and incorporates radiology (in the wider sense), nuclear medicine, investigative endoscopy, radiological sciences, medical photography, (medical) thermography, and microscopy (e.g. for human pathological investigations). Primarily designed Measurement and recording techniques which are not supposed to produce images, such, magneto encephalography (MEG), as electroencephalography (EEG), electrocardiography (ECG), and others, can be seen as forms of medical imaging because they produce data susceptible to be represented as maps (i.e., containing positional information)..Mathematical models are the basic foundation of biomedical computing. Fundamental technique for achieving scientific progress in experimental, biomedical, clinical and behavioral research is creating models on the basis of data extracted from images. As compared to the visible light photographs and microscope primates of the early 20th century, now day's medical images are acquired by a range of techniques across all biological scales which are far more sophisticated than earlier techniques. The geometrically arranged arrays of data samples may be considered to be modern medical images which quantify such diverse physical phenomena as the diffusion of water molecules through and within tissue or as the time variation of hemoglobin deoxygenate ion during neuronal metabolism.

A key research area is the formulation of biomedical engineering principles based on rigorous mathematical foundations in order to develop general-purpose software methods that can be integrated into complete therapy delivery systems. Such systems support the more efficient delivery of many image-guided processes such as minimally invasive surgery, biopsy, and radiation therapy. We focus our analysis on four main parts of image-guided therapy (IGT) and image-guided surgery (IGS) so as to understand the extensive role of imaging in the therapeutic process, and to acknowledge the current usage of images before, during, and after treatment and these are localization, targeting, monitoring, and control. [29]

\subsection{Some of Medical Imaging techniques: \\ a. Ultrasonography \\ b. Computed Tomography \\ c. Magnetic Resonance Imaging \\ d. Positron Emission Tomography}

\section{LITERATURE REVIEW}

Seungwoo Ryu et all “ Evaluation of Dielectric Resonator Sensor for Near-Field Breast Tumor Detection" 2011 IEEE In this paper, an H-shape ultra wide band (UWB) dielectric resonator (DR) mounted on a vertical ground plane edge with broadside radiation patterns is evaluated for breast tumor detection. In order to scan the whole breast, the proposed DR sensor can be attached to the skin without a need for matching medium. The sensor has a wide half power beam width, therefore path loss is reduced. Accurate tumor response is obtained due to the compact size and very good sensor characteristics. 
Koley et all "Brain MRI Segmentation for Tumor Detection using Cohesion based Self Merging Algorithm" 2011 IEEE In this paper, presented a segmentation of brain MRI for the purpose of determining the exact location of brain tumor using CSM based partitional $\mathrm{K}$ means clustering algorithm. This approach is much simpler and computationally less complex and computation time is very less.

Zhang et all "A novel automatic tumor detection for breast cancer ultrasound Images" 2011 IEEE

In this paper, proposed a novel automatic method for breast ultrasound (BUS) image tumor detection. Here used the fuzzy logic theory and transform an US image into fuzzy domain. An iterative method is used to find threshold. The proposed approach can automatically detect the tumor in BUS image with high accuracy.

Selvam et all "Brain Tumor Detection using Scalp EEG with Modified Wavelet-ICA and Multi Layer Feed Forward Neural Network" 2011 IEEE.This paper presents a method of detecting the brain tumor using the first, second and third order statistics of the scalp EEG with a Modified Wavelet-Independent Component Analysis (MwICA) technique and a multi-layer feedforward neural network.

Unde et all "Novel Edge Detection Approach on Active Contour for Tumor Segmentation" 2012 IEEE In this paper, proposed new method of edge detection for active contours based on local adaptive threshold technique via variational energy minimization to stop the contour at desired object boundary. By using proposed method, can control the evaluation of curve with moderate value of $\alpha$ for all images.

Lewis et all"Detection of Breast Tumor Candidates Using Marker-controlled Watershed Segmentation and Morphological Analysis" 2012 IEEE In this paper, a marker-controlled watershed segmentation algorithm was developed to locate breast mass tumor candidates. The approach first selected foreground and background markers, and then applied watershed segmentation algorithm to isolate a tumor region from its surrounding tissue. Since watershed segmentation is based on pixel density variation that is present in all mass tumors, the proposed approach was fairly successful in locating tumors under all conditions.

selva kumar et all“ Brain Tumor Segmentation and Its Area Calculation in Brain MR Images using K-Mean Clustering and Fuzzy C-Mean Algorithm" 2012 IEEE This project uses computer aided method for segmentation (detection) of brain tumor based on the combination of two algorithms. This method allows the segmentation of tumor tissue with accuracy and reproducibility comparable to manual segmentation. In addition, it also reduces the time for analysis. At the end of the process the tumor is extracted from the MR image and its exact position and the shape also determined. The stage of the tumor is displayed based on the amount of area calculated from the cluster.

Jiang et all"Learning-Based Automatic Breast Tumor Detection And Segmentation In Ultrasound Images"
2012 IEEE In this paper, proposed a novel algorithm for automatic detection of breast tumors in US images. Formulated the tumor detection as a two step learning problem: tumor localization by bounding box and exact boundary delineation. Specifically, the proposed method uses an Ada Boost classifier on Harr-like features to detect a preliminary set of tumor regions. The preliminarily detected tumor regions are further screened with a support vector machine using quantized intensity features.

Parisot et all "Graph-based Detection, Segmentation \& Characterization of Brain Tumors" 2012 IEEE In this paper proposed a novel approach for detection, segmentation and characterization of brain tumors. This method exploits prior knowledge in the form of a sparse graph representing the expected spatial positions of tumor classes. Such information is coupled with image based classification techniques along with spatial smoothness constraints towards producing a reliable detection map with in a unified graphical model formulation. Towards optimal use of prior knowledge, a two layer interconnected graph is considered with one layer corresponding to the low-grade glioma type (characterization) and the second layer to voxel-based decisions of tumor presence.

\section{ANT COLONY OPTIMIZATION}

The brief history of the ant colony optimization metaheuristic is mainly a history of experimental research. Trial and error guided all early researchers and still guides most of the ongoing research efforts. This is the typical situation for virtually all existing metaheuristics: it is only after experimental work has shown the practical interest of a novel metaheuristic that researchers try to deepen their understanding of the metaheuristic's functioning not only through more and more sophisticated experiments but also by means of an effort to build a theory. Typically, the first theoretical problem considered is the one concerning convergence: will the metaheuristic

Find the optimal solution if given enough resources? Other questions that are often investigated are the speed of convergence, principled ways of setting the metaheuristic's parameters, relations to existing approaches, identification of problem characteristics that make the metaheuristic more likely to be successful, understanding the importance of the different metaheuristic components, and so on. In this chapter we address those problems for which we have an answer at the time of writing. In particular, we discuss the convergence of some types of ACO algorithms to the optimal solution and the relationship between ACO and other well-known techniques such as stochastic gradient ascent.

Theoretical Considerations on ACO When trying to prove theoretical properties for the ACO metaheuristic, the researcher faces a first major problem: ACO's very general definition. Although generality is a desirable property - it allows putting in the same framework antbased algorithms applied to discrete optimization problems that range from static problems such as the traveling salesman problem to time-varying problems such as routing in telecommunications networks -it makes 
theoretical analysis much more complicated, if not impossible.

Ant colony algorithm was first proposed by Dorigo et al. as a multi agent solution to optimization problems such as travelling salesperson. This algorithm is inspired by studies and researches on ant colonies. Studies show that ants are social insects which live in colonies, and tend to survive the colony rather than surviving individuals. One of the most amazing behaviors about ants is how they find food and especially how they find the shortest path between the food source and the nest. This behavior is a kind of mass intelligence (using different intelligent agent) which has recently been the matter of interest to scientists. Ants deposit a special chemical substance named

Pheromone on their way to find food. The amount of pheromone depends on the length of the path and the quality of the food. Other ants smell the pheromone and are attracted by this path and they also amplify the pheromone on this path. Shorter paths get more pheromone and so, the more pheromone there is, and the more ants tend to pass along the path. Thus the shortest paths are chosen by ants. Although the pheromone evaporates in a short time, it remains as an ant's trace for a limited time. Ants' movement is based on a simple instinctive behavior. They choose the path which has more pheromone or in other words, they choose the path from which more ants have passed. The important point is that, although choosing the path with more pheromone is more probable, it is not deterministic. So probability and stochasticity play an important role in ant colony algorithm. Another point is the evaporation of pheromone left on the path. By passing time, more pheromone evaporates and probability of choosing a certain path is decreased.

Actually ant colony algorithm is a heuristic method for solving problems through making a graph. A lot of ants start to move on the problem solution space and each individual plays its small part to solve the problem [21]. Choosing a direction by an ant depends on the amount of pheromone on the path and also the heuristic function. In order to solve the problem, ant $\mathrm{k}$ uses the probabilistic selection rule to choose a path. Probability of $\mathrm{k}^{\text {th }}$ ant's movement from $\mathrm{I}$ to $\mathrm{j}$ can be calculated by

$$
P_{i j}^{k}=\frac{\left[\tau_{i j}\right]^{\alpha}\left[\eta_{i j}\right]^{\beta}}{\sum_{l \in N_{i}^{k}}\left[\tau_{i l}\right]^{\alpha}\left[\eta_{i l}\right]^{\beta}} \text {, if } j \in N_{i}^{k}
$$

Where $\tau_{\mathrm{ij}}$ is the amount of deposited pheromone between nodes $\mathrm{I}$ and $\mathrm{j}$ and also $\mathrm{N}_{\mathrm{i}}^{\mathrm{k}}$ is the neighborhood nodes for ant $\mathrm{k}$ in the node $\mathrm{i}$. This neighborhood has some nodes that have not been yet seen by the ant $\mathrm{k}$. By using a technique of making a black list called Tabu, we can prevent an ant to choose a duplicate node. Constants aand $\beta$ will control the influence of pheromone and heuristic function. Finally $\mathrm{N}_{\mathrm{i}}^{\mathrm{k}}$ is the value of heuristic function. After completing the path, the pheromone amount on the path will be updated by

$$
\tau_{i j}^{\text {new }}=\tau_{i j}^{\text {current }}+\sum_{k=1}^{m} \Delta \tau_{i j}^{k}
$$

Where $\Delta \tau_{\mathrm{ij}}{ }^{\mathrm{k}}$ is the pheromone amount which is added to the previous one by ant $\mathrm{k}$ to go from $\mathrm{I}$ to $\mathrm{j} . \mathrm{m}$ is the total number of ants.

\section{RESULTS}

We are using Brain MRi as a input images for detecting and locating the brain tumor in the brain. .These images are as shown below.
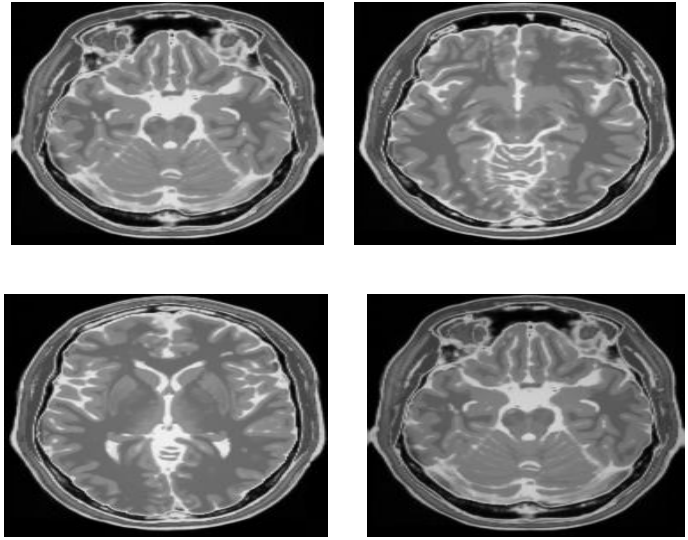

Fig 1:The different MRI images of brain

These are the different database images which are suffering from brain tumor.
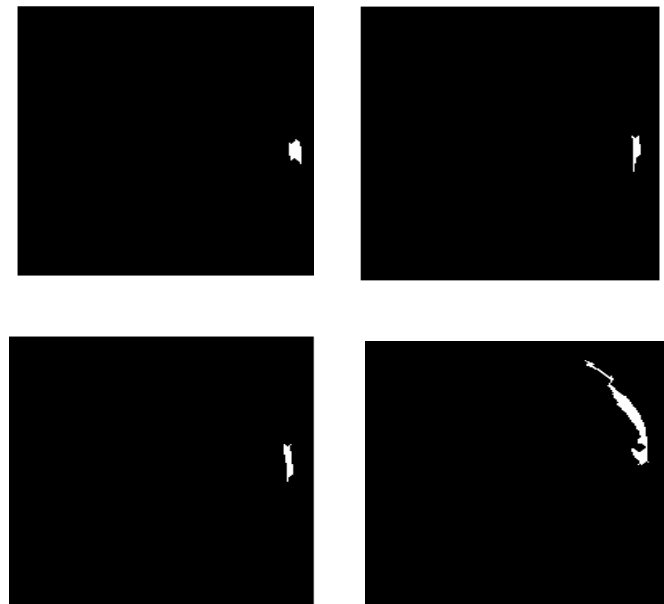

Figure 2: predicted area of tumor in MRI's images

Step by step images during algorithm process are given below:

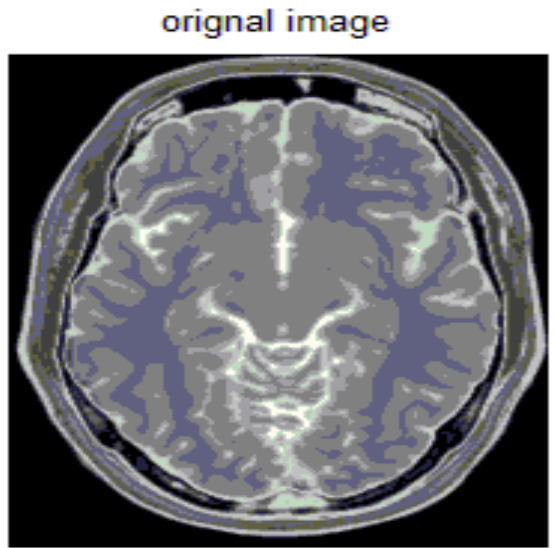

Figure 3: original image 
INTERNATIONAL JOURNAL OF INNOVATIVE RESEARCH IN ELECTRICAL, ELECTRONICS, INSTRUMENTATION AND CONTROL ENGINEERING Vol. 4, Issue 2, February 2016

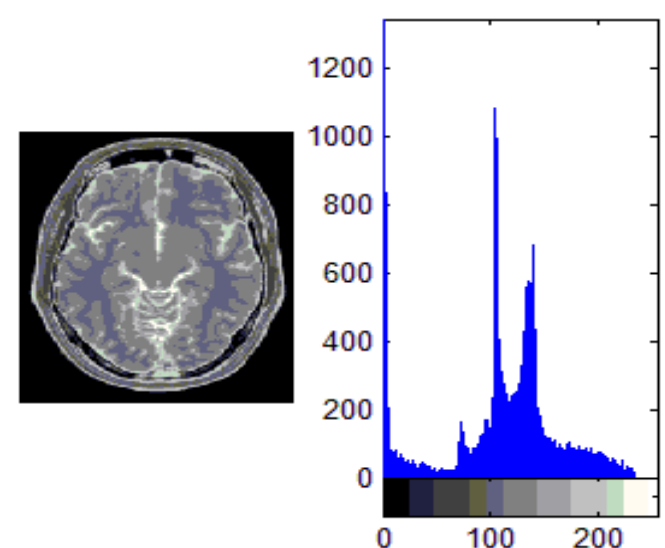

Figure 5.4 original image before histogram equalization

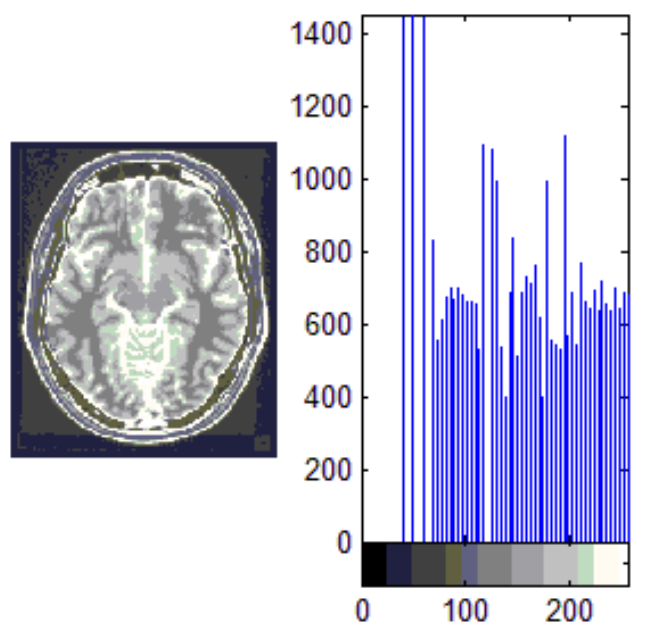

Figure 5.5: image after histrogram equalization

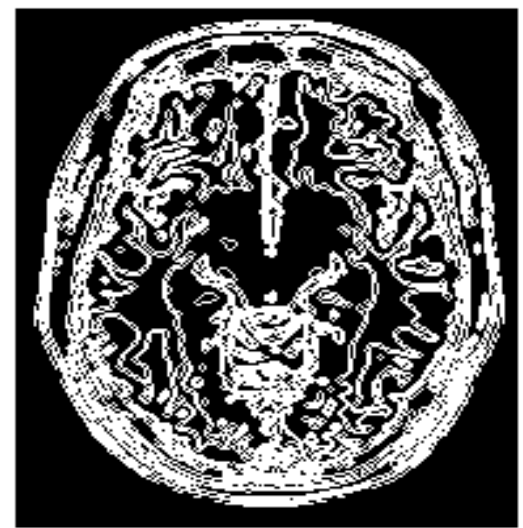

Figure 5.6: image segmentation with multi thresholding

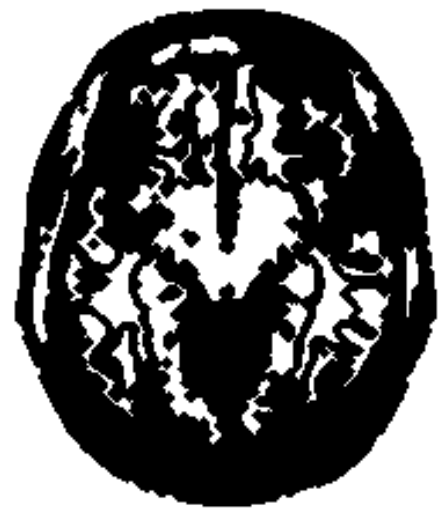

Figure 5.7: To overcome from oversegmentation

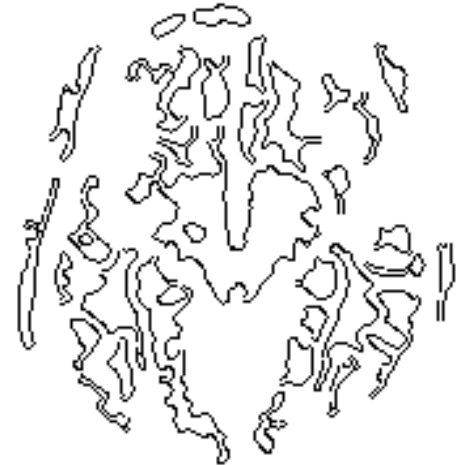

Figure 5.8: observing different objects

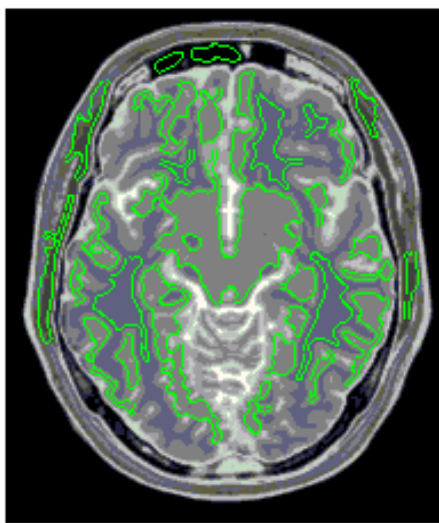

Figure 5.9: marking different objects of brain

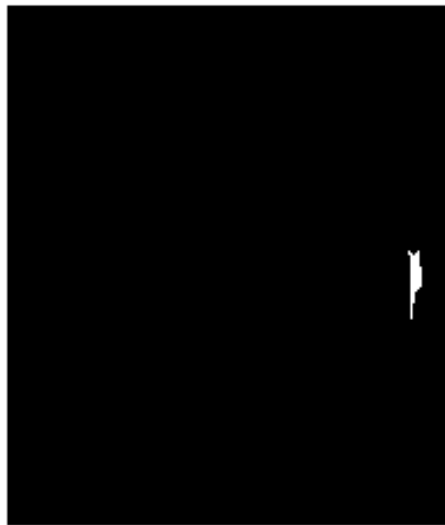

Figure5.10: detecting tumor location in brain

Parameter Analyzed (Sensitivity, Specificity \& Accuracy)

Table 1: Parameter calculation of the different MRI's

\begin{tabular}{|c|c|c|c|}
\hline MRI Image no & Sensitivity & Specificity & Accuracy \\
\hline MRI Image 1 & 65.7459 & 100 & 99.857 \\
\hline MRI Image 2 & 87.963 & 100 & 99.97 \\
\hline MRI Image 3 & 88.8889 & 100 & 99.9677 \\
\hline MRI Image 4 & 97.8355 & 100 & 99.9769 \\
\hline MRI Image 5 & 89.5349 & 100 & 99.9792 \\
\hline MRI Image 6 & 97.3684 & 100 & 99.9908 \\
\hline MRI Image 7 & 98.324 & 100 & 99.9792 \\
\hline MRI Image 8 & 91.129 & 100 & 99.9746 \\
\hline MRI Image 9 & 96.2366 & 100 & 99.9331 \\
\hline MRI Image 10 & 99.4169 & 100 & 99.9954 \\
\hline Average & $94.32 \%$ & 100 & $99.97 \%$ \\
\hline
\end{tabular}


ACO, is implemented on the different MRI images, and it provide $98.89 \%$ accuracy and $94.32 \%$ sensitivity and specificity $100 \%$.the Table 5.2 represents the parameter of the different images

\section{CONCLUSION}

In this thesis, proposed new method to detect tumor using Ant Colony Optimization (ACO) to optimize our area of detection. Multi thresh holding is used to stop the contour at desired object boundary to overcome over segmentation problem. The proposed method is more efficient and is less error sensitive. the effectiveness of the proposed method is well demonstrated by the experimental result. In future, we will explore more advanced classification techniques and contour segmentation strategies.

\section{REFERENCES}

[1] Adam Santorelli, Emily Porter, Milica Popović , Joshua Schwartz "Pulse Shaping for Time-Domain Microwave Breast Tumour Detection: Experiments with Realistic Tissue Phantoms" 2011 IEEE

[2] Alejandro Garcia-Uribe, Cheng-Chung Chang, Murat Kaya Yapici, Jun Zou, Bhaskar Banerjee, John Kuczynski, Evan S. Ong, Erin S. Marner, and Lihong V. Wang "High-Transmission-Efficiency and Side-Viewing Micro OIDRS Probe for Fast and Minimally Invasive Tumor Margin Detection" 2010 IEEE

[3] Amit Satish Unde, Premprakash V. A., Praveen Sankaran "Novel Edge Detection Approach on Active Contour for Tumor Segmentation" 2012 IEEE

[4] Daniel Greenwald, Caroline G.L. Cao and Emily W. Bushnell "Haptic Detection of Artificial Tumors by Hand and with a Tool in a MIS Environment" 2012 IEEE

[5] J.selva kumar, A.Lakshmi, T.Arivoli “ Brain Tumor Segmentation and Its Area Calculation in Brain MR Images using K-Mean Clustering and Fuzzy C-Mean Algorithm" 2012 IEEE

[6] Kenny Seungwoo Ryu and Ahmed A. Kishk "Evaluation of Dielectric Resonator Sensor for Near-Field Breast Tumor Detection" 2011 IEEE

[7] K. S. Angel Viji , Dr J. Jayakumari "Automatic Detection of Brain Tumor based on Magnetic Resonance Image using CAD System with watershed segmentation" 2011 IEEE

[8] Ling Zhang, Chencheng Huang, Yuping Ren, Fushan Liu "A novel automatic tumor detection for breast cancer ultrasound Images" 2011 IEEE

[9] Ms. M. Parisa Beham , Ms.A.B.Gurulakshmi "MORPHOLOGICAL IMAGE PROCESSING APPROACH ON THE DETECTION OF TUMOR AND CANCER CELLS"

[10] Peng Jiang, Jingliang Peng,Guoquan Zhang, Erkang Cheng,Vasileios Megalooikonomou, Haibin Ling "Learning-Based Automatic Breast Tumor Detection And Segmentation In Ultrasound Images" 2012 IEEE

[11] Sarah Parisot, Hugues Duffau, Stephane Chemouny, Nikos Paragios "Graph-based Detection, Segmentation \& Characterization of Brain Tumors" 2012 IEEE

[12] Subhranil Koley and Aurpan Majumder "Brain MRI Segmentation for Tumor Detection using Cohesion based Self Merging Algorithm" 2011 IEEE

[13] V. Salai Selvam and S. Shenbagadevi "Brain Tumor Detection using Scalp EEG with Modified Wavelet-ICA and Multi Layer Feed Forward Neural Network" 2011 IEEE. 